\title{
In memory of Stanislav Julianovich Sila-Novitsky
}

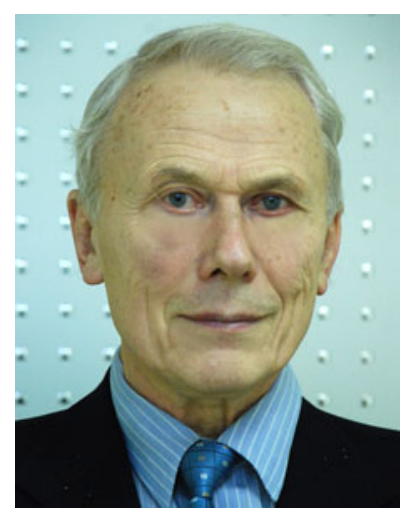

With great sadness, we note that Stanislav Julianovich SilaNovitsky (Stas) unexpectedly passed away at age of 72 on March 7, 2010, due to natural causes. He has been an active member of the GPS Solution Editorial Board since 1998.

This exceptional scientist began his carrier with a MSEE degree from the Control and Communication RadioSystems subdivision, Radio-electronics department of Moscow Aviation Institute, in 1961 and continued postgraduate school education.

From 1964 until 1993, Stas worked for the Russian (former Soviet Union) various research and design institutes of space industry instruments (NIITP, NIIP, RNIIKP).
He pioneered research on digital processing of radio signals for the Russian space industry. Due to his efforts, an international rescue Cospas-Sarsat system was based on digital algorithms.

He led research and designed algorithms and software in areas for navigation of aircrafts equipped with synthetic aperture antenna and the first GLONASS digital receiver.

Starting in 1993, he served as general manager of the Russian branch of Ashtech, a US company owned by Dr. Javad Asjaee, where he directed the development of a series of pioneering high precision GPS/GLONASS commercial receivers. In recent years, he had been a general manager of Javad GNSS assisting in building the satellite navigation industry.

Stanislav Sila-Nivitsky, who was the author and co-author of numerous books, papers, and patents, was a real icon in the space navigation industry. His expert knowledge was invaluable to GPS Solutions.

Stas will always be remembered by beloved relatives and grateful colleagues.

Alfred Leick

Editor In Chief

GPS Solutions 